\title{
Improving lung cancer survival; time to move on
}

\author{
Marlies E Heuvers ${ }^{1 *}$, Joost P Hegmans ${ }^{1}$, Bruno H Stricker ${ }^{2,3,4}$ and Joachim G Aerts ${ }^{1,5}$
}

\begin{abstract}
Background: During the past decades, numerous efforts have been made to decrease the death rate among lung cancer patients. Nonetheless, the improvement in long-term survival has been limited and lung cancer is still a devastating disease.

Discussion: With this article we would like to point out that survival of lung cancer could be strongly improved by controlling two pivotal prognostic factors: stage and treatment. This is corresponding with recent reports that show a decrease in lung cancer mortality by screening programs. In addition, modulation of the patient's immune system by immunotherapy either as monotherapy or combined with conventional cancer treatments offers the prospect of tailoring treatments much more precisely and has also been shown to lead to a better response to treatment and overall survival of non-small cell lung cancer patients.

Summary: Since only small improvements in survival can be expected in advanced disease with the use of conventional therapies, more research should be focused on lung cancer screening programs and patient tailored immunotherapy with or without conventional therapies. If these approaches are clinically combined in a standard multidisciplinary policy we might be able to advance the survival of patients with lung cancer.
\end{abstract}

Keywords: Lung cancer, Survival, Lung cancer screening, Immunotherapy

\section{Background}

Lung cancer is the leading cause of cancer-related death worldwide. Approximately $85 \%$ of all cases of lung cancer are non-small cell lung cancer (NSCLC). The 5-year survival of this aggressive disease is only 16\% [1]. One of the reasons for this extremely poor survival is that most lung cancer cases are diagnosed at an advanced stage due to the relative lack of clinical symptoms during early stages. Metastatic NSCLC is currently an incurable disease for which standard chemotherapy provides only minor improvement in overall survival. In addition, less than $30 \%$ of patients with advanced-stage NSCLC have a response to platinum-based chemotherapy, the most commonly used first line treatment at this stage of the disease [2].

During the last decades, advances in diagnostic and therapeutic approaches of this devastating disease have been made, however, long-term survival rates have hardly changed in the past 50 years [3]. Therefore, new approaches are required.

\footnotetext{
* Correspondence: m.heuvers@erasmusmc.nl

${ }^{1}$ Department of Respiratory Diseases and Tuberculosis, Erasmus Medical University Center, Rotterdam, The Netherlands

Full list of author information is available at the end of the article
}

\section{Discussion}

Survival of lung cancer could be strongly improved by controlling two pivotal prognostic factors: stage and treatment. Early stages of lung cancer have a better prognosis; thus early diagnosis of lung cancer by screening programs is one way that leads to a reduction in lung cancer mortality. However, given the high chance of tumor recurrence, even alleged early stage NSCLC patients with adequate surgical resection can have undetectable metastases at diagnosis $[4,5]$. It is known that adjuvant chemotherapy can reduce these metastases; nevertheless, in $24 \%$ of the patients metastasis occurs after adjuvant chemotherapy [4]. Therefore, besides lung cancer screening programs, an additional approach next to the conventional therapy must be developed to tackle lung cancer. In recent years it has been established that the immune system plays an important role in carcinogenesis and makes an essential contribution to the anti-tumor effects of traditional therapies. Modulation of the patient's immune system by immunotherapy either as monotherapy or combined with conventional cancer treatments offers the prospect of tailoring treatments much more precisely and could lead to a better response to treatment and overall survival of NSCLC patients.

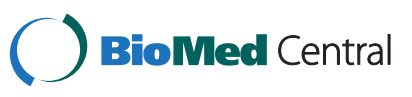

(c) 2012 Heuvers et al.; licensee BioMed Central Ltd. This is an Open Access article distributed under the terms of the Creative Commons Attribution License (http://creativecommons.org/licenses/by/2.0), which permits unrestricted use, distribution, and reproduction in any medium, provided the original work is properly cited. 
Taken together, when early diagnosis by screening programs and patient-tailored immunotherapy are combined in a standard multidisciplinary policy for NSCLC treatment, we might be able to advance the survival of patients with early stage lung cancer. We will discuss both topics and their role in improving lung cancer survival below.

\section{Lung cancer screening}

Multiple randomized trials have investigated the effectiveness of lung cancer screening and it is shown that lung cancer can be identified at an early stage with detection rates varying between 40-66\% [6,7]. The survival rates of lung cancer patients diagnosed in screening programs are very high; 5- and even 10-year survival rates close to $90 \%$ can be achieved $[8,9]$. The largest lung cancer screening trial [10] recently showed that screening of high risk persons is very effective in reducing the mortality from lung cancer. Persons with more than 30 pack-years (PY) and aged between 55 and 74 years at time of randomization were included in this study. They found a relative mortality reduction of $20 \%$ when this high-risk group is screened with a low-dose computer tomography (CT) scan compared to chest radiography [10]. However, this is probably an underestimate, as the mortality reduction was measured at the time of closure of the trial. The introduction of lowdose multi-detector CT has led to important advantages, such as advanced scan speed, better spatial resolution, and the capacity to reconstruct multiple series from a single data acquisition. Before public policy recommendations are crafted, there are major concerns in lung cancer screening such as the effects of false positive findings, lead-time bias, the impact of overdiagnosis, and the generalizability of the results [11].

Another important aspect that should be considered in generalizing the results of screening studies are the therapeutic options for patients with a positive screening, as lung cancer treatment is an important prognostic factor. In developed countries, lung cancer patients are treated with surgery, chemotherapy, and radiotherapy. In recent years, peri-operative mortality has decreased by the introduction of video assisted thoracoscopy (VATS) and better perioperative management [12]. Early stage patients who are not eligible for surgery are frequently treated with radiotherapy with curative intent. Novel radiotherapy techniques, such as stereotactic ablative radiotherapy, show local control rates of $90 \%$ or more for stage I NSCLC [13]. Adjuvant chemotherapeutic regimens have been shown to increase survival especially in resected patients with stage II and IIIA disease [14]. These regimens are expensive and therefore the results of the published screening trials can only be applied to the selected group of individuals in countries with well-developed health care systems with a quality comparable to the US.

\section{Adjuvant immunotherapy}

Treatment of lung cancer is currently based on the patient's clinical signs and symptoms, tumor stage and subtype, medical history, and data from imaging and laboratory evaluation. Until now, most cancer research is focused on therapies based on tumor characteristics to improve the prognosis of NSCLC, as cancer has long been considered as a cell-autonomous genetic disease. However, the sobering outcome of current NSCLC therapy has shifted the attention to combining adjuvant treatment approaches.

Recent experimental findings and clinical observations have led to cancer-related immune inflammation being acknowledged as a new hallmark of cancer [15-17]. Evidence that the immune system of the host can influence cancer incidence, cancer growth, response to therapy, and the prognosis of the disease, is growing [18]. Therefore it was thought that conventional therapy combined with immunotherapy based on a pretreatment profile of the immune system of the host could be a valuable tool to increase the survival of early stage NSCLC [19].

Cancer immunotherapy attempts to activate the host's immune system to recognize and destroy the residual lung cancer cells that conventional therapy misses. Immunotherapy can be divided into two main types: passive and active immunotherapy [20,21]. The most common form of passive immunotherapy is monoclonal antibody therapy [20]. It makes use of antibodies that have been produced in vitro and can bind to specific cell surface proteins that can influence tumor growth [22]. However, there will only be a response of the immune system during the time the antibody is present in the body. Ipilimumab (anti-CTLA-4), bevacizumab (anti-VEGF), and anti programmed death (anti-PD-1) or anti-PD ligand 1 (Anti-PD-L1) are examples of passive immunotherapy that could be useful in NSCLC [23-26].

Ipilimumab blocks the negative cytotoxic T-lymphocyte antigen (CTLA)-4 that enhances T-cell responses to tumor cells, leading to effective immune responses. For NSCLC, ipilimumab is now in phase II development [24], but it is already approved by the US Food and Drug Administration (FDA) for the treatment of unresectable or metastatic melanoma $[24,27,28]$. Studies show that the twoand three-year survival rates in ipilimumab-containing treatment arms in metastatic melanoma patients are almost twice as high as in the non-ipilimumab-containing treatment arm.

Bevacizumab is an antibody that neutralizes the vascular endothelial growth factor (VEGF) ligand. As a result, it will inhibit angiogenesis [29]. Moreover, research has shown that adding bevacizumab to chemotherapy is associated with afferent vascular dilatation and efferent vascular constriction of tumor vessels that may help concentrate chemotherapy at the tumor site. Bevacizumab combined with taxane-platinum chemotherapy is the first approved 
antiangiogenic agent for cancer therapy that showed increase of progression-free survival and overall survival in first-line treatment of stage IV NSCLC $[29,30]$. Recently, data have been published on the immunogenic effect of VEGF. VEGF seems to be involved in a number of mechanisms negatively influencing the immune system; it makes dendritic cells more tolerogenic, and induces myeloid derived suppressor cells. Adding bevacizumab prevents immunotolerance and could thereby contribute to a better survival of lung cancer [31,32].

Two other recently described antibodies that could play important roles in passive immunotherapy are anti-PD-1 and anti-PD-L1 [25,26]. PD-1 is a co-inhibitory receptor on activated T-cells that plays an important role in immunosuppression. PD-L1, the ligand of PD-1, is expressed on cancer cells and is involved in negative regulation of immune responses, as they increase apoptosis of T-cells and inhibit CD4 and CD8 T-cell activation [25,26]. Inhibition of the interaction between PD-1 and PD-L1 can improve T-cell responses and mediate antitumor activity. Recent studies show that in NSCLC the objective response rates to anti-PD-1 and anti-PD-L1 are 18\% and 10\% respectively $[25,26]$. Blockage of both receptors induced durable tumor regression and prolonged stabilization of the disease. These findings confirm that the pathway between PD1 and PD-L1 could play an important role in therapeutic intervention and that it causes an increase in survival of lung cancer patients.

Active immunotherapy tries to persuade and boost immune effector cells in vivo against tumor cells through the administration of immune mediators capable of activating the humoral (antibodies) and cellular ( $\mathrm{T}$ cells) immune system [33]. Therefore the duration of this broad response persists for a long time, because of the immunologic memory and it is less prone to antigen mutational responses [33]. Currently, multiple trials are investigating the effectiveness of different lung cancer vaccines [33-36]. In 2001, one of the first synthetic lung cancer vaccines showed that 16 out of 65 patients had an immune response after vaccination, and the median survival time was more than doubled (30.6 months, instead of 13.3 months in controls) [37]. After that, other tumor-antigens vaccines, such as Wilms tumor antigen-1 and IDM-2101 were tested and showed immunological responses and prolonged survival in patients with lung cancer $[21,36]$. Next to synthetic vaccines there are trials that test dendritic cell (DC) vaccines $[34,38,39]$. In DC vaccines, tumor associated antigens are used to load immature autologous DCs. These DCs are injected into patients to stimulate antigen-specific immune responses in lung cancer patients. Different studies have shown biological activity of DC vaccines and phase I and II trials report that a group of lung cancer patients had therapeutic benefit $[34,39,40]$. Nevertheless, until now, reports about clinical applicability are anecdotal.
Other examples of active immunotherapy in lung cancer are natural killer (NK) cell transfer and adoptive $\mathrm{T}$ cell transfer $[41,42]$.

As described above, recent literature provides evidence for many potentially useful immunotherapy combinations. However, these therapies show drastic antitumor responses in only small subsets of patients. Currently, there is lack of predictive biomarkers to rationally choose combinations of immunotherapy for individual patients that benefit from these therapies. Therefore, it is necessary to further elucidate the mechanisms that are responsible for clinical benefit in small groups of patients and identify relevant pre-treatment biomarkers that distinguish responders from non-responders. This patient-tailored treatment approach is able to redress the balance towards efficacious antitumor responses that can improve the overall survival for more patients.

Taken together, passive and active immunotherapy might have an important adjuvant role in early stage NSCLC by consolidating responses to conventional therapy and thereby leading to increased lung cancer survival rates. However, further research in this field is warranted to improve these therapies and to define subsets of responders.

\section{Summary}

During the past decades, numerous efforts have been made to decrease the death rate among lung cancer patients. Nonetheless, the improvement in long-term survival has been limited and lung cancer is still a devastating disease [3].

Since only small improvements in survival can be expected in advanced disease with the use of conventional therapies, more research should be focused on early stage lung cancer. Combining lung cancer screening programs and patient tailored immunotherapy with or without conventional therapies should be further explored. If these approaches are clinically combined in a standard multidisciplinary policy we might be able to advance the survival of patients with lung cancer.

\section{Abbreviations}

NSCLC: Non-small cell lung cancer; PY: Pack-years; CT: Computer tomography; VATS: Video assisted thoracoscopy; FDA: US food and drug administration; VEGF: Vascular endothelial growth factor; DC: Dendritic cell; NK cell: Natural killer cell.

\section{Competing interests}

The authors declare that they have no competing interests.

\section{Authors' contributions}

All authors were major contributors in writing the manuscript. All authors read and approved the final manuscript.

\section{Author details}

${ }^{1}$ Department of Respiratory Diseases and Tuberculosis, Erasmus Medical University Center, Rotterdam, The Netherlands. ${ }^{2}$ Department of Epidemiology, Erasmus Medical University Center, Rotterdam, The 
Netherlands. ${ }^{3}$ Department of Internal Medicine, Erasmus Medical University Center, Rotterdam, The Netherlands. ${ }^{4}$ Department of Medical Informatics, Erasmus Medical University Center, Rotterdam, The Netherlands. ${ }^{5}$ Department of Respiratory Diseases and Tuberculosis, Amphia Hospital, Breda, The Netherlands.

Received: 27 September 2012 Accepted: 29 November 2012 Published: 13 December 2012

\section{References}

1. Siegel R, Ward E, Brawley O, Jemal A: Cancer statistics, 2011: the impact of eliminating socioeconomic and racial disparities on premature cancer deaths. CA Cancer J Clin 2011, 61:212-236.

2. Schiller $\mathrm{JH}$, et al: Comparison of four chemotherapy regimens for advanced non-small-cell lung cancer. N Engl J Med 2002, 346:92-98.

3. Sugimura $\mathrm{H}$, Yang P: Long-term survivorship in lung cancer: a review. Chest 2006, 129:1088-1097.

4. Goodgame B, et al: Risk of recurrence of resected stage I non-small cell lung cancer in elderly patients as compared with younger patients. J Thorac Oncol 2009, 4:1370-1374.

5. Goodgame B, et al: A clinical model to estimate recurrence risk in resected stage I non-small cell lung cancer. Am J Clin Oncol 2008, 31:22-28

6. Blanchon T, et al: Baseline results of the Depiscan study: a French randomized pilot trial of lung cancer screening comparing low dose CT scan (LDCT) and chest X-ray (CXR). Lung Cancer 2007, 58:50-58.

7. van Klaveren RJ, et al: Management of lung nodules detected by volume CT scanning. N Engl J Med 2009, 361:2221-2229.

8. International Early Lung Cancer Action Program, et al: Survival of patients with stage I lung cancer detected on CT screening. N Engl J Med 2006, 355:1763-1771.

9. Vansteenkiste J, Dooms C, Mascaux C, Nackaerts K: Screening and early detection of lung cancer. Ann Oncol 2012, 23(Suppl 10):x320-x327.

10. National Lung Screening Trial Research, T, et al: Reduced lung-cancer mortality with low-dose computed tomographic screening N Engl J Med 2011, 365:395-409.

11. Heuvers ME, Stricker BH, Aerts JG: Generalizing lung-cancer screening results. N Engl J Med 2012, 366:192-193.

12. Whitson BA, Groth SS, Duval SJ, Swanson SJ, Maddaus MA: Surgery for early-stage non-small cell lung cancer: a systematic review of the video-assisted thoracoscopic surgery versus thoracotomy approaches to lobectomy. Ann Thorac Surg 2008, 86:2008-2016. discussion 2016-2008.

13. Dahele M, Senan S: The role of stereotactic ablative radiotherapy for early-stage and oligometastatic non-small cell lung cancer: evidence for changing paradigms. Cancer Res Treat 2011, 43:75-82.

14. Wisnivesky JP, et al: Survival and risk of adverse events in older patients receiving postoperative adjuvant chemotherapy for resected stages II-IIIA lung cancer: observational cohort study. BMJ 2011, 343:d4013.

15. Heuvers ME, et al: History of tuberculosis as an independent prognostic factor for lung cancer survival. Lung Cancer 2012, 76:452-456.

16. Cavallo F, De Giovanni C, Nanni P, Forni G, Lollini PL: 2011: the immune hallmarks of cancer. Cancer Immunol Immunother 2011, 60:319-326.

17. Hanahan D, Weinberg RA: Hallmarks of cancer: the next generation. Cell 2011, 144:646-674.

18. Caux C, Zitvogel L: Recent successes of cancer immunotherapy: a new dimension in personalized medicine? Target Oncol 2012, 7:1-2.

19. Zitvogel $L$, et al: Integration of host-related signatures with cancer cell-derived predictors for the optimal management of anticancer chemotherapy. Cancer Res 2010, 70:9538-9543.

20. Keyler DE, et al: Monoclonal nicotine-specific antibodies reduce nicotine distribution to brain in rats: dose- and affinity-response relationships. Drug Metab Dispos 2005, 33:1056-1061.

21. Jadus MR, et al: Lung cancer: a classic example of tumor escape and progression while providing opportunities for immunological intervention. Clin Dev Immunol 2012, 2012:160724.

22. Sharma P, Wagner K, Wolchok JD, Allison JP: Novel cancer immunotherapy agents with survival benefit: recent successes and next steps. Nat Rev Cancer 2011, 11:805-812

23. Cornelissen $\mathrm{R}$, et al: New roads open up for implementing immunotherapy in mesothelioma. Clin Dev Immunol 2012, 2012:927240.
24. Cameron F, Whiteside G, Perry C: Ipilimumab: first global approval. Drugs 2011, 71:1093-1104

25. Brahmer JR, et al: Safety and activity of anti-PD-L1 antibody in patients with advanced cancer. N Engl J Med 2012, 366:2455-2465.

26. Topalian SL, et al: Safety, activity, and immune correlates of anti-PD-1 antibody in cancer. N Engl J Med 2012, 366:2443-2454.

27. Sondak VK, Smalley KS, Kudchadkar R, Grippon S, Kirkpatrick P: Ipilimumab. Nat Rev Drug Discov 2011, 10:411-412

28. Minchom A, Young K, Larkin J: Ipilimumab: showing survival benefit in metastatic melanoma. Future Oncol 2011, 7:1255-1264.

29. Dillman RO: Cancer immunotherapy. Cancer Biother Radiopharm 2011, 26:1-64.

30. Claret $\mathrm{L}$, et al: Simulations using a drug-disease modeling framework and phase ii data predict phase III survival outcome in first-line non-smallcell lung cancer. Clin Pharmacol Ther 2012, 92:631-634.

31. Manzoni M, et al: Immunological effects of bevacizumab-based treatment in metastatic colorectal cancer. Oncology 2010, 79:187-196.

32. Osada T, et al: The effect of anti-VEGF therapy on immature myeloid cell and dendritic cells in cancer patients. Cancer Immunol Immunother 2008, 57:1115-1124

33. Winter $H$, et al: Active-specific immunotherapy for non-small cell lung cancer. J Thorac Dis 2011, 3:105-114.

34. Um SJ, et al: Phase I study of autologous dendritic cell tumor vaccine in patients with non-small cell lung cancer. Lung Cancer 2010, 70:188-194.

35. Neninger Vinageras $\mathrm{E}$, et al: Phase II randomized controlled trial of an epidermal growth factor vaccine in advanced non-small-cell lung cancer. J Clin Oncol 2008, 26:1452-1458.

36. Barve $M$, et al: Induction of immune responses and clinical efficacy in a phase II trial of IDM-2101, a 10-epitope cytotoxic T-lymphocyte vaccine, in metastatic non-small-cell lung cancer. J Clin Oncol 2008, 26:4418-4425.

37. Palmer M, et al: Phase I study of the BLP25 (MUC1 peptide) liposomal vaccine for active specific immunotherapy in stage IIIB/IV non-small-cell lung cance. Clin Lung Cancer 2001, 3:49-57. discussion 58.

38. Hegmans JP, et al: Consolidative dendritic cell-based immunotherapy elicits cytotoxicity against malignant mesothelioma. Am J Respir Crit Care Med 2010, 181:1383-1390.

39. Hirschowitz EA, Foody T, Hidalgo GE, Yannelli JR: Immunization of NSCLC patients with antigen-pulsed immature autologous dendritic cells. Lung Cancer 2007, 57:365-372.

40. Perroud MW Jr, et al: Mature autologous dendritic cell vaccines in advanced non-small cell lung cancer: a phase I pilot study. J Exp Clin Cancer Res 2011, 30:65.

41. Iliopoulou EG, et al: A phase I trial of adoptive transfer of allogeneic natural killer cells in patients with advanced non-small cell lung cancer. Cancer Immunol Immunother 2010, 59:1781-1789.

42. Rosenberg SA, Restifo NP, Yang JC, Morgan RA, Dudley ME: Adoptive cell transfer: a clinical path to effective cancer immunotherapy. Nat Rev Cancer 2008, 8:299-308.

doi:10.1186/1471-2466-12-77

Cite this article as: Heuvers et al:: Improving lung cancer survival; time to move on. BMC Pulmonary Medicine 2012 12:77.

\section{Submit your next manuscript to BioMed Central and take full advantage of:}

- Convenient online submission

- Thorough peer review

- No space constraints or color figure charges

- Immediate publication on acceptance

- Inclusion in PubMed, CAS, Scopus and Google Scholar

- Research which is freely available for redistribution 